\title{
Formation professionnelle continue pour les médecins militaires
}

Adrian Leutenegger

Prof. Dr, doyen du ASMC

Correspondance:

LBA - Sanität, Direktion SAMK

Worblentalstrasse 36

CH-3063 Ittigen

Tél. 0313242760

Fax 0313237197

info-samk@vtg.admin.ch
Le projet d'Académie suisse de médecine militaire et de catastrophe (ASMC) est devenu entre temps une institution bien établie de l'armée et du Service sanitaire coordonné (SSC).

En 2010 également, l'ASMC offre aux médecins militaires un large éventail de cours de perfectionnement et de séances, qu'ils pourront suivre dans le cadre des obligations militaires, individuellement et comme service soldé. L'offre de l'ASMC comprend aussi bien des cours de spécialistes de l'armée (C spéc) que des séances et des cours de diverses sociétés spécialisées, dont la fréquentation est financée par l'ASMC. Parmi cela, on trouve de nombreux cours qui doivent être suivis pour l'obtention de divers titres de médecins spécialistes.

Kursangebote der SAMK / Offres des cours ASMC 2010

* = bereits ausgebucht/occupés

Anästhesie und Reanimation in Basel

Kurssprache deutsch

Anästhesiekongress (englisch)

26./27.3.2010 BS 1

Dienstarztkurs (DAK)

19./20.8. + 16./17.9.2010 BS 2

ACLS (Advanced Cardiovascular Life Support)*

ATLS (Advanced Trauma Life Support)*

29./30.4.2010 BS 3

PALS (Pediatric Advanced Life Support)

TPAM (Training in Prehospital Airway Management)

In französischer Sprache

$\begin{array}{lll}\text { TPAM (Training in Prehospital Airway Management) } & 6.5 .2010 & \text { BS } 7\end{array}$

TPAM (Training in Prehospital Airway Management)

Sanitätsdienstliche Führung Grossereignis (SFG)

(Teil A: «Knowledge», Grundlagen, Prinzipien, Partner.

Voraussetzungen: Notarzt SGNOR oder Rettungssanitäter

in Kaderposition (oder äquivalent). (deutsch)

Sanitätsdienstliche Führung Grossereignis (SFG)

(Teil B: «Training», Kommunikation und

24./25.11.2010

BS SFG-B

Teaminteraktion, deutsch). Voraussetzung: SFG-A

Innere Medizin, Infektiologie in Bern

32. Infektiologie-Symposium, Luzern

22.4.2010

BE 1A

3. Kombiniertes Infektiologie-Curriculum und -Symposium

11.11 .2010

21.10.2010

BE $1 B$

9. Berner Notfall-Symposium

22.10.2010

BE $2 A$

5. Systematisches Curriculum, internmedizinische Notfallmedizin

ACLS Kurs (Basel)*

27./28.4.2010

BE 3

ACLS Kurs (Basel)* ACLS Kurs (Zürich)*

29./30.4.2010

10./11.6.2010

BE 4

ACLS Kurs (Bern)*

22./23.6.2010

BE 5

ACLS Kurs (Bern)*
BE 6

BE 7 
Kursangebote der SAMK / Offres des cours ASMC 2010

* = bereits ausgebucht/occupés

Innere Medizin, Infektiologie in Bern

Dienstarztkurs SGNOR, Zürich*

20./21.5+ 3./4.6.2010 BE 8

Dienstarztkurs SGNOR, Basel

19./20.8+ 16./17.9.2010 BE 9

Weiter- und Fortbildungskurs der SGIM und

17./18. 3.2010

BE10

Innere Medizin in Praxis und Klinik

Médecine et management en cas de catastrophe et de guerre, à Lausanne

Cours en langue française

Principes de médecine de catastrophe

21./22.1.2010

VD $1[\mathrm{M} 1]$

(risques, concepts d'intervention, terminologie)

Accident majeur, cours pour professionnels de la chaîne des urgences

$24 .-26.6 .2010$

VD 2 [M2]

Accident majeur, cours de conduite pour médecins-chefs des secours

9./10.9.2010

VD $3[\mathrm{M} 3]$

et ambulanciers chef des secours.

Plan catastrophe et gestion de crise à l'hôpital

18./19.11.2010

VD $6[\mathrm{M} 6]$

Formation pré-requise: VD 1 [M1]

ATLS (Advanced Trauma Life Support)*

6.-8.9.2010 VD 7

ACLS (Advanced Cardiovascular Life Support)*

27./28.4.2010

VD 8

ACLS (Advanced Cardiovascular Life Support)

29./30.4.2010

VD 9

ACLS (Advanced Cardiovascular Life Support)

4./5.11.2010

VD 10

PALS (Pediatric Advanced Life Support)

21./22.6.2010

VD 11

PALS (Pediatric Advanced Life Support)*

8./ 9.11 .2010

VD 12

PALS (Pediatric Advanced Life Support)

11./12.11.2010

VD 13

Cours de médicine d'urgence (SSMUS)

21.-24. 9.2010

VD 14

Chirurgie à Genève

Cours de chirurgie de guerre et de catastrophe (théorie) (französisch)

9./10.9.2010

GE 1

Cours de chirurgie de guerre et de catastrophe (pratique)

20.4.2010

GE 2

Katastrophen- und Wehrpsychiatrie in Zürich

** Die Kurse ZH 3 bis ZH 9 können nur im Paket zusammen mit den Kursen ZH 1 oder ZH 2 gebucht werden

Kernkompetenz Stressmanagement:

25./26.3.10

ZH 1

Umgang mit Belastungen in der Akutmedizin.

Kernkompetenz Stressmanagement:

16./17.9.10

ZH 2

Umgang mit Belastungen in der Akutmedizin.

ACLS (Advanced Cardiovascular Life Support)*

10./11.6.210

$\mathrm{ZH} 3^{* *}$

ACLS (Advanced Cardiovascular Life Support)*

26./27.8.2010

$\mathrm{ZH} 4$ **

ACLS (Advanced Cardiovascular Life Support)

21./22.10.2010

$\mathrm{ZH} 5^{* *}$

ATLS (Advanced Trauma Life Support)*

17./18.6.2010

$\mathrm{ZH} 6^{* *}$

ATLS (Advanced Trauma Life Support)*

21./22.6.2010

$\mathrm{ZH} 7^{* *}$

ATLS (Advanced Trauma Life Support)*

13./14.9.2010

$\mathrm{ZH} 8^{* *}$

PHTLS-Provider

20./21.8.2010

$\mathrm{ZH} 9^{* *}$

Fachzentrum für Zahnmedizin und Kieferchirurgie, Basel

SAKM (Schweizerische Arbeitsgemeinschaft für

Kieferchirurgie und Mundkrankheiten)

6. Bernd Spiessl Symposium für innovative und

17.- 19.6 .2010

ZM 2

visionäre Technologien

Fachzentrum für Transfusionswesen

12. Weiterbildungstagung und Symposium BSD SRK/SVTM in Interlaken

2.-4.9.2010

TW 1

Fachzentrum Medizinische B-Abwehr, Zürich

Kriterien und Vorbereitung von Auslandeinsätzen

30.9.2010

MBA 2

Kursanmeldungen ausschliesslich elektronisch ab Homepage!

http://cmsr.intranet.lba.admin.ch/internet/lba/de/home/themen/sanit/neu/neu2/Formular2010.html 